\title{
TRATAMIENTO ANAEROBIO DEL SUERO DE QUESO EN REACTORES DISCONTINUOS Y UASB A ESCALA LABORATORIO
}

\author{
ANAEROBIC TREATMENT OF CHEESE WHEY IN BATCH AND UASB REACTORS AT \\ LABORATORY SCALE
}

${ }^{1}$ Deyanira Liviac Calderon y ${ }^{2}$ Rosemary Vela Cardich

\begin{abstract}
Resumen
Se evaluó a escala laboratorio la biodegradabilidad anaerobia del suero de queso en reactores discontinuos y la eficiencia del reactor de flujo ascendente en manto de lodos (UASB) para reducir la demanda química de oxígeno (DQO) del suero de queso. Se utilizó como sustrato el suero producido en la elaboración de queso fresco en la Planta Piloto de Leche (PPL) de la Universidad Nacional Agraria La Molina (UNALM). La biodegradación anaerobia del suero de queso $\left(32-35^{\circ} \mathrm{C}\right)$ con concentraciones alrededor de $4 \mathrm{~g} \mathrm{DQO} / \mathrm{L}$ no presentó inhibición; por el contrario, sí lo hicieron muestras de suero con concentraciones de 41 y 75 g DQO/L. Este comportamiento se ha asociado con la presencia de altas concentraciones de sodio en las soluciones de suero más concentradas. El reactor UASB demostró ser un eficiente método de tratamiento de suero de queso diluido $\left(35^{\circ} \mathrm{C}\right)$ para la reducción de DQO. Se obtuvieron porcentajes de remoción promedio de DQO entre 77 y $86 \%$, trabajando con tiempos de residencia hidráulicos (TRHs) entre 0,45 y 1,75 días, velocidades de carga orgánica (VCOs) entre 2,5 y 7,9 g DQO/L.d y concentraciones de DQO en la alimentación entre 1,1 y $12,1 \mathrm{~g}$ DQO/L. La adición de alcalinidad al suero de queso fue fundamental para alcanzar un pH óptimo y la estabilidad del sistema.
\end{abstract}

Palabras clave: biodegradabilidad, suero de queso, tratamiento, reactor UASB.

\begin{abstract}
Anaerobic biodegradability of cheese whey in batch reactors and cheese whey COD removal efficiency of an upflow anaerobic sludge blanket (UASB) reactor were evaluated at laboratory scale. It was used as substrate, whey generated during cheese-making at Milk Pilot Plant (PPL) of La Molina National Agrarian University (UNALM). Anaerobic biodegradation of cheese whey $\left(32-35^{\circ} \mathrm{C}\right)$ with concentrations around $4 \mathrm{~g}$ COD/L showed no inhibition; on the contrary, cheese whey samples with concentrations of 41 and $75 \mathrm{~g} \mathrm{COD} / \mathrm{L}$ did so. This result has been associated with the presence of high concentrations of sodium in the more concentrated cheese whey solutions. The UASB reactor proved to be an efficient method of treatment of diluted cheese whey $\left(35^{\circ} \mathrm{C}\right)$ for removing COD. COD average removals of 77 to $86 \%$ were obtained working with hydraulic retention times between 0,45 to 1,75 days, organic loading rates between 2,5 and 7,9 g COD/L.d and influent COD between 1.1 and $12.1 \mathrm{~g} \mathrm{COD/L.} \mathrm{Alkalinity} \mathrm{addition} \mathrm{to} \mathrm{cheese} \mathrm{whey} \mathrm{was}$ essential for optimum $\mathrm{pH}$ and stability of the system.
\end{abstract}

Key words: biodegradability, cheese whey, treatment, UASB reactor.

\section{Introducción}

En las zonas urbanas del Perú sólo aproximadamente el $48 \%$ de las aguas residuales reciben tratamiento (Superintendencia Nacional de Servicios de Saneamiento - SUNASS, 2013) y lo restante es vertido al mar, ríos o lagos. La situación es más crítica en las zonas rurales, donde el porcentaje de efluentes tratados alcanza valores aún menores.
Entre las fuentes de contaminación del agua se encuentran algunos efluentes agroindustriales como los de la industria láctea. Esta industria genera efluentes con características muy variadas, que dependen del tipo de producto procesado (leche yogurt, queso, etc.), del método de operación, entre otros factores (Demirel B., Yenigun O., Onay T., 2005). Un rango que con frecuencia se encuentra en la literatura para la concentración de DQO en estas aguas residuales es de 1 a 9 g/L (Demirel et al., 2005), lo que se debe principalmente a la presencia de suero de queso. El suero contiene entre 60 y $80 \mathrm{~g}$

\footnotetext{
Departamento Ing. Ambiental Fisica y Meteorologia, Facultad de Ciencias, UNALM, Lima, Perú. E-mail: dliviac@lamolina.edu.pe

${ }^{2}$ Departamento Dpto. Ing. Ambiental Fisica y Meteorologia, Facultad de Ciencias, UNALM, Lima, Perú. E-mail: rvela@lamolina.edu.pe
} 
DQO/L (Mockaitis G., Ratusznei, S., Rodrigues J., Zaiat M., Foresti E., 2006), y no sólo es importante como fuente contaminante por esta característica sino también por el volumen generado, que es alrededor de un 80 a $90 \%$ del volumen de la leche procesada (Mockaitis et al., 2006).

El suero es rico en proteínas, sin embargo, el uso de tecnologías de valorización pueden hacer antieconómico el re-uso del suero de queso, en particular en industrias lácteas de mediano y pequeño tamaño (Rico C., Muñoz N., Rico J.L., 2015). Cuando los costos asociados a las tecnologías de valorización son altos, la disposición del suero de queso se convierte en un problema ambiental (Rico et al., 2015).

El proceso de digestión anaerobia es un tipo de tratamiento particularmente atractivo para residuos con alta carga orgánica, tal como el suero de queso (Dareioti M., Dokianakis S., Stamatelatou K, Zafiri C., Kornaros M., 2009), debido a que no requiere de oxígeno y necesita poco espacio, por lo que presenta un bajo costo; genera poca cantidad de lodo en exceso (Demirel et al., 2005) y permite la recuperación de cantidades significativas de energía en forma de gas metano (Ergüder T., Tezel U., Güven E., Demirer G., 2001).

El reactor anaerobio de tipo UASB ha sido empleado exitosamente para el tratamiento de las aguas residuales lácteas a distintas escalas durante dos décadas (Demirel et al., 2005), principalmente en los países europeos. Se han tratado efluentes en un rango de concentraciones que oscilan entre los 12 y 60 g DQO/L, con porcentajes de remoción de carga orgánica de 85 a $99 \%$, aplicando un TRH de 6 días y VCO en el rango de 2 a 7,3 g DQO/ L.d (Demirel et al., 2005). No obstante, también se ha reportado que altas concentraciones de sustrato afectan el buen desempeño del reactor UASB (Yan J., Lo K., Liao P., 1989) y que el suministro de nutrientes, metales traza y/o alcalinidad es vital para el tratamiento del suero de queso (Ergüder et al., 2001).

Yan et al. (1989), observaron problemas de estabilidad en un reactor UASB de laboratorio que trabajó con suero de queso con una DQO de $38 \mathrm{~g} / \mathrm{L}$. El problema se atribuyó a la acumulación de ácidos grasos volátiles (AGV), llegando a la conclusión de que el proceso UASB es limitado en términos de tiempo de retención y que el sistema no tolera un efluente lácteo muy concentrado.

Con el fin de seguir investigando respecto a la degradación anaerobia del suero de queso y su posible aplicación al tratamiento de las aguas residuales de la industria láctea en el Perú, el presente trabajo tuvo como objetivos evaluar a escala de laboratorio la biodegradabilidad anaerobia del suero de queso en reactores discontinuos y la eficiencia del reactor UASB para reducir la DQO del suero de queso, para ello se utilizó como sustrato el suero producido en la elaboración de queso fresco en la Planta Piloto de Leche (PPL) de la Universidad Nacional Agraria La Molina (UNALM).

\section{Materiales y Métodos}

Caracterización del suero de queso de la planta piloto de leche

En el proceso de desuerado de queso fresco de la PPL de la UNALM, se generan 3 tipos de suero. El primer suero es el que presenta mayor DQO. El segundo, tiene una cantidad de DQO ligeramente menor que el primer suero, porque es diluido con agua. Finalmente el tercer suero presenta la menor cantidad de carga orgánica y a diferencia de los otros dos, contiene una elevada concentración de sodio.

La muestra de suero que se caracterizó consistió en una mezcla de $35 \%$, $45 \%$ y $20 \%$ del 1er, 2 do y 3 er suero, respectivamente.Los parámetros analizados, fueron, $\mathrm{pH}$, DQO, fósforo total, nitrógeno total, sólidos totales, sólidos suspendidos totales, aceites y grasas, calcio, magnesio, sodio, sulfato, alcalinidad total y temperatura. Para la determinación de éstos parámetros se utilizaron los métodos estándar (American Public Health Association - APHA, 2005), excepto para los parámetros nitrógeno total y aceites y grasas, que se analizaron usando los métodos oficiales de análisis (Association of official Analytical chemists - AOAC, 1998).

Los análisis se llevaron a cabo en el Laboratorio de Ingeniería Ambiental, Laboratorio de Química y Laboratorio de Suelos de la UNALM.

\section{Determinación de la actividad metanogénica de los lodos anaerobios}

Los lodos anaerobios que se inocularon en las pruebas de biodegradabilidad del suero de queso y en el reactor UASB se caracterizaron a partir de la actividad metanogénica específica máxima, según una adaptación del método propuesto por (Soto M., Méndez R., Lema J., 1992). El equipo para la determinación de la actividad metanogénica del lodo anaerobio se representa en la Fig. 1. El digestor tiene un volumen de $500 \mathrm{ml}$ y se conecta a un Frasco de Mariotte que contiene una solución de hidróxido de sodio de $25 \mathrm{~g} / \mathrm{L}$. La concentración de lodo en el digestor, medida como sólidos volátiles (SV), es $5 \mathrm{~g}$ $\mathrm{SV} / \mathrm{L}$. El sustrato consiste en una solución de ácido acético con una DQO de $2 \mathrm{~g} / \mathrm{L}$. Se adiciona $\mathrm{Na} 2 \mathrm{~S}$ como reductor y NaHCO3 como fuente de alcalinidad. Finalmente, se ajusta el pH a 7,0 añadiendo $\mathrm{NaOH}$.

El biogás generado es recogido en el Frasco de Mariotte y el volumen de solución desplazada por el metano es medido en una probeta. Los ensayos se realizaron a temperatura ambiente promedio de $20^{\circ} \mathrm{C}$. La determinación de sólidos volátiles se realizó siguiendo el método Standard (APHA, 2005). 


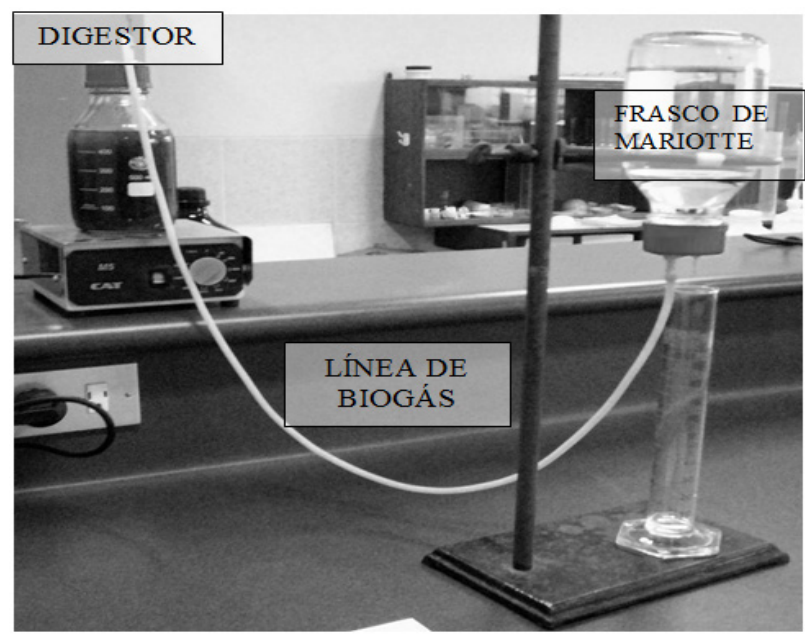

Figura 1. Equipo para la determinación de la actividad metanogénica del lodo anaerobio.

Fuente: Elaboración Propia

\section{Determinación de la biodegradabilidad anaerobia del suero de queso}

Las pruebas de biodegradabilidad anaerobia se llevaron a cabo según una adaptación del método propuesto por Soto et al. (1992) y con el uso de micronutrientes, de acuerdo a una metodología adaptada de Ergüder et al. (2001). Se utilizaron digestores sin agitación conteniendo $100 \mathrm{~mL}$ de soluciones de suero de distintas concentraciones. Se trabajó en un rango de temperatura entre 32 y $35^{\circ} \mathrm{C}$. La concentración de lodo fue $2 \mathrm{~g} \mathrm{SV} / \mathrm{L}$. El lodo anaerobio que se usó como inoculo procedía de un reactor de laboratorio empleado para el tratamiento de aguamiel del café.

En una primera etapa se trabajó con 2 series de 4 digestores cada una. La primera serie sin agregar micronutrientes y la segunda con adición de micronutrientes, los cuales fueron $\mathrm{CuCl} 2(0,4 \mathrm{mg} / \mathrm{L}), \mathrm{ZnCl} 2(0,5 \mathrm{mg} / \mathrm{L}), \mathrm{AlCl} 3.6 \mathrm{H} 2 \mathrm{O}$ $(0,5 \mathrm{mg} / \mathrm{L}), \mathrm{NaMoO} 4.2 \mathrm{H} 2 \mathrm{O}(0,5 \mathrm{mg} / \mathrm{L}), \mathrm{NiCl} 2.6 \mathrm{H} 2 \mathrm{O}$ $(0,5 \mathrm{mg} / \mathrm{L}), \mathrm{NaWO} 4.2 \mathrm{H} 2 \mathrm{O}(0,5 \mathrm{mg} / \mathrm{L})$ y $\mathrm{Na} 2 \mathrm{SeO} 3(0,5$ $\mathrm{mg} / \mathrm{L}$ ). Ambas series trabajaron con sulfuro de sodio $(100 \mathrm{mg} / \mathrm{L})$, bicarbonato de sodio $(1 \mathrm{~g} / \mathrm{L})$ e hidróxido de sodio hasta $\mathrm{pH}$ neutro. Las concentraciones de suero utilizadas en cada serie fueron de 0 (control), 5, 50 y $90 \%$ en volumen. La duración de esta 1ra etapa fue 50 días.La segunda etapa se diseñó a partir de los resultados obtenidos en la primera etapa y su duración fue 39 días. Como medida de la biodegradabilidad anaerobia se calculó el porcentaje de metanización usando la siguiente ecuación (Soto et al., 1992):

$$
\% \mathrm{M}=\frac{\left(\mathrm{DQO}_{\mathrm{CH} 4}\right) \mathrm{e}}{\left(\mathrm{DQO}_{\mathrm{i}}\right)} * 100
$$

donde:

$\left(\mathrm{DQO}_{\mathrm{CH} 4}\right) \mathrm{e}$ : DQO del metano del biogás a la temperatura del sistema.

$\mathrm{DQO}_{\mathrm{i}}$ : DQO inicial estimada en base al resultado de la caracterización del suero.

149

\section{Implementación, operación y monitoreo del reactor UASB a escala de laboratorio}

El reactor UASB tenía un volumen de 14,75 L y fue inoculado con una mezcla, en igual proporción, de lodo anaerobio procedente de una planta de tratamiento de aguas residuales cerveceras y lodo de un reactor anaerobio de laboratorio que trataba aguamiel de café. En la base del reactor se puso una cama de piedras, encima se colocó $4.5 \mathrm{~L}$ de lodo anaerobio ( $30 \%$ del volumen total del reactor).

Durante la operación, la alimentación que se mantenía agitada y a temperatura ambiente, junto con la recirculación, eran impulsadas por una bomba peristáltica hacia la zona de ingreso ubicada cerca a la base del reactor. La recirculación de una parte del efluente tratado se hizo para favorecer la mezcla dentro del reactor. La temperatura dentro del reactor se mantuvo entre 34,5 y $35,5^{\circ} \mathrm{C}$.

El suero que se utilizó para preparar la alimentación del reactor era una mezcla del 1er, 2 do y $3 \mathrm{er}$ desuerado de la PPL en una proporción del 35\%, 45\% y $20 \%$, respectivamente. Una vez preparada la mezcla, se realizó un pretratamiento que consistió en una acidificación natural durante una semana (en un recipiente cerrado) y a temperatura ambiente, luego se retiró la capa de grasa formada en la parte superior.

La alimentación del reactor consistió en suero diluido con agua potable. Además, se adicionó micronutrientes, de acuerdo con los resultados obtenidos en la prueba de biodegradabilidad del suero, y se neutralizó con $\mathrm{NaHCO}_{3}$ e $\mathrm{NaOH}$.

La concentración del suero lácteo de la alimentación y el TRH fueron incrementados sucesivamente en las 4 etapas de experimentación, así la VCO también varió. La Tabla 1 resume las condiciones de los parámetros de operación de cada etapa de trabajo.

Tabla 1. Parámetros de operación del reactor UASB durante a experimentación.

\begin{tabular}{|c|c|c|c|c|}
\hline \multirow{2}{*}{ Parámetro } & \multicolumn{4}{|c|}{ Etapas } \\
\hline & 1 & 2 & 3 & 4 \\
\hline $\begin{array}{l}\text { Tiempo del } \\
\text { ensayo (d) }\end{array}$ & $0-90$ & $91-125$ & $126-153$ & $154-209$ \\
\hline $\begin{array}{c}\text { DQO } \\
\text { alimentación } \\
(\mathrm{mg} / \mathrm{L})\end{array}$ & $492-1780$ & $2020-2895$ & $6110-7420$ & $6350-12090$ \\
\hline TRH (d) & $0,42-0,52$ & $0,71-0,97$ & $1,52-1,89$ & $1,40-1,67$ \\
\hline $\begin{array}{l}\mathrm{VCO}(\mathrm{g} \\
\mathrm{DQO} / \mathrm{L} . \mathrm{d})\end{array}$ & $0,96-4,26$ & $2,24-4,05$ & $3,24-4,88$ & $4,15-8,62$ \\
\hline
\end{tabular}

Cada etapa de experimentación se finalizó cuando el coeficiente de variabilidad de las eficiencias de remoción 
de DQO se encontraba dentro del rango de 0 a 15 , es decir con calificación de muy homogéneo u homogéneo (Mendenhall W., Beaver R., Beaver B., 2006).

El pH de la alimentación se midió diariamente. La alcalinidad al bicarbonato de la alimentación y del efluente, se determinaron dos veces por semana.

La alcalinidad al bicarbonato se estimó a partir de la alcalinidad parcial (PA) usando el método propuesto por (Ripley L., Boyle W., Converse J., 1986). Este método consiste en titular la muestra con ácido fuerte hasta $\mathrm{pH} 5,75$ porque a tal $\mathrm{pH}$ aproximadamente el $80 \%$ del ión bicarbonato es valorado (Jenkins, S.; Morgan, J. y Sawyer, C., 1983). El método busca reducir la titulación de los ácidos grasos cuya capacidad buffer para mantener el $\mathrm{pH}$ cercano a la neutralidad es nula. A las condiciones del análisis menos del $20 \%$ de los ácidos volátiles es valorado (Jenkins et al. 1983).

También se determinó la relación entre la alcalinidad intermedia (IA) y la alcalinidad parcial (PA) del efluente estudiada por Ripley et al. (1986). El valor de la relación IA/PA se incrementa rápido cuando existe un problema de digestión y luego decrece cuando se recupera el sistema. La IA se determina titulando la muestra desde pH 5,75 hasta $\mathrm{pH} \mathrm{4,3}$ y corresponde de manera aproximada a la alcalinidad de los ácidos volátiles (Ripley et al. 1986). En el presente trabajo la relación IA/PA se ha representado como un índice $\alpha$.

Durante la experimentación se determinaron una vez por semana, como mínimo, la DQO de la alimentación y la del efluente. Con los resultados se calcularon las eficiencias de remoción de DQO.

\section{Resultados y discusión}

\section{Caracterización del suero de queso de la PPL de la UNALM}

Los resultados de la caracterización del suero de queso se presentan en la tabla 2. La relación DQO/N/P cumplió con la relación mínima de 350/5/1 para sistemas anaerobios (Chernicharo, 2007). También se constató la presencia de otros nutrientes importantes para el tratamiento anaerobio, tales como calcio y magnesio. Por otro lado, se observó que las concentraciones de sodio y cloruros eran elevadas.

Tabla 2. Caracterización del suero de queso de la Planta Piloto de Leche.

\begin{tabular}{ccc}
\hline Parámetro & Unidad & Valor \\
\hline $\mathrm{pH}$ & Unidades de $\mathrm{pH}$ & 6,59 \\
$\mathrm{DQO}$ & $\mathrm{g} / \mathrm{L}$ & 82,83 \\
Fósforo Total & $\mathrm{mg} \mathrm{P} / \mathrm{L}$ & 82 \\
Nitrógeno Total & $\mathrm{g} / 100 \mathrm{~g} \mathrm{de} \mathrm{muestra}$ & 0,13 \\
\hline
\end{tabular}

Continuación de tabla

\begin{tabular}{ccc}
\hline Parámetro & Unidad & Valor \\
\hline Sólidos Totales & $\mathrm{g} / \mathrm{L}$ & 49,72 \\
Sólidos Volátiles & $\mathrm{g} / \mathrm{L}$ & 37,96 \\
Sólidos Suspendidos & $\mathrm{g} / \mathrm{L}$ & 2,67 \\
Totales & & 0,5 \\
Aceites y grasas & $\mathrm{g} / 100 \mathrm{~g}$ de muestra & 375 \\
Calcio & $\mathrm{mg} / \mathrm{L}$ & 95 \\
Magnesio & $\mathrm{mg} / \mathrm{L}$ & 163 \\
Potasio & $\mathrm{mg} / \mathrm{L}$ & 3800 \\
Sodio & $\mathrm{mg} / \mathrm{L}$ & 649,92 \\
Sulfatos & $\mathrm{mg} / \mathrm{L}$ & 5857,5 \\
Cloruros & $\mathrm{mg} / \mathrm{L}$ & 0,58 \\
Boro & $\mathrm{mg} / \mathrm{L}$ & 841 \\
\hline Alcalinidad Total & $\mathrm{mg} / \mathrm{L}$ & \\
\hline
\end{tabular}

Fuente: Elaboración propia

\section{Pruebas de biodegradabilidad anaerobia}

El lodo que se usó como inóculo durante las pruebas de biodegradabilidad anaerobia presentó una concentración de 51,4 g SV/L y una actividad metanogénica de $0,3 \mathrm{~g}$ DQO/g SV.d.

En la tabla 3 se muestran los valores iniciales de DQO calculados para las diferentes soluciones de suero en cada serie de las pruebas de biodegradabilidad anaerobia. Estos valores se han calculado a partir del dato de DQO obtenido en la caracterización del suero de queso (Tabla 2).

Tabla 3.Valores calculados de DQO de las soluciones de suero en las pruebas de biodegradabilidad anaerobia.

\begin{tabular}{ccccc}
\hline \multirow{2}{*}{ Parámetro } & \multirow{2}{*}{ Unidad } & \multicolumn{3}{c}{ Valores calculados de DQO } \\
\cline { 3 - 5 } & & $5 \%$ & $50 \%$ & $90 \%$ \\
\hline DQO & $\mathrm{g} / \mathrm{L}$ & 4 & 41 & 75 \\
\hline
\end{tabular}

Fuente: Elaboración Propia

En la primera etapa de los ensayos sólo se obtuvieron resultados positivos para la solución al $5 \%$ en suero porque los ensayos al $50 \%$ y $90 \%$ presentaron acidificación por insuficiente alcalinidad. En la Fig. 2 se muestra la producción acumulada de metano en función del tiempo cuando se trabajó con una concentración al $5 \%$ en suero. Se observa que el uso de micronutrientes no mejoró la producción de metano a lo largo del tiempo. De hecho, los porcentajes de metanización en ambas experiencias al $5 \%$, con micronutrientes y sin ellos, fue aproximadamente $100 \%$, lo que se desprende de la Tabla 4 donde se presentan los porcentajes de metanización obtenidos en cada serie de ensayos. El resultado de $107 \%$ para la prueba sin micronutrientes puede atribuirse al error que se comete por la estimación del valor de la DQO inicial. 
Los resultados alcanzados al 5\% están de acuerdo con los reportados por Ergüder et al. (2001), quienes trabajando con una solución de suero de queso con $5.5 \mathrm{~g} \mathrm{DQO} / \mathrm{L}$, obtuvieron porcentajes de metanización mayores al $90 \%$ en ensayos con micronutrientes.

Figura 2. Producción acumulada de metano vs tiempo para: solución de suero al 5\% con micronutrientes ( $\downarrow)$, solución de suero al 5\% sin micronutrientes (घ), Control.

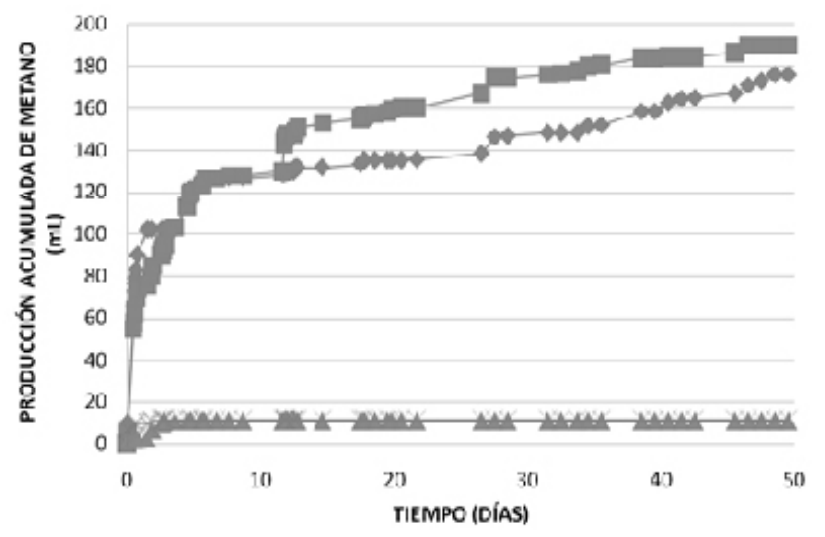

.con micronutrientes $(\boldsymbol{\Delta})$, Control sin micronutrientes (X).

Fuente: Elaboración propia

Tabla 4. Porcentajes de metanización de los ensayos al 5, 50 y $90 \%$ de suero con y sin micronutrientes.

\begin{tabular}{ccc}
\hline $\begin{array}{c}\text { Concentración } \\
\text { de suero }\end{array}$ & $\begin{array}{c}\% \text { de } \\
\text { metanización } \\
\text { sin } \\
\text { micronutrientes }\end{array}$ & $\begin{array}{c}\% \text { de } \\
\text { metanización } \\
\text { con } \\
\text { micronutrientes }\end{array}$ \\
\hline $5 \%$ & 107 & 99 \\
$50 \%$ & 26 & 42 \\
$90 \%$ & 13 & 19 \\
\hline
\end{tabular}

Fuente: Elaboración Propia

En la segunda etapa de los ensayos de biodegradabilidad se repitieron las pruebas al 50\% y 90\%. En esta ocasión las proporciones utilizadas de DQO, SV, NaHCO3 y micronutrientes fueron las que se usaron en el ensayo al 5\% en suero, es decir: 0,5 g SV/g DQO, 0,25 g $\mathrm{NaHCO} 3 / \mathrm{g}$ DQO y una relación promedio de $0,125 \mathrm{mg}$ de micronutrientes/g DQO.

En la Fig. 3 se presenta la producción acumulada de metano en función del tiempo para concentraciones al $50 \%$ y $90 \%$ de suero. Se observa que el uso de micronutrientes favoreció la producción de metano. En estos casos los porcentajes de metanización fueron mayores en los ensayos con micronutrientes (Tabla 4), aún así los valores fueron bajos, llegando sólo al 42\% y $19 \%$, respectivamente.

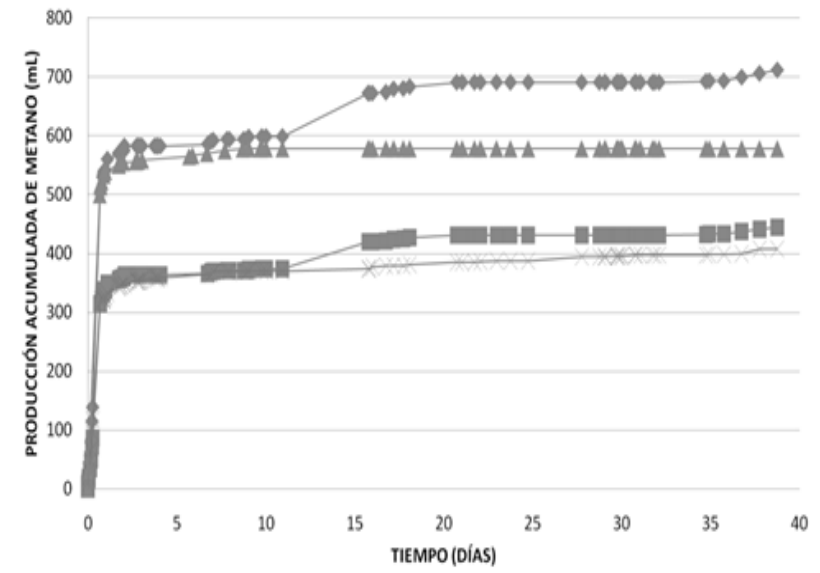

Figura 3. Producción acumulada de metano vs tiempo para:solución de suero al $50 \%$ sin micronutrientes (匹), Solución de suero al $50 \%$ con micronutrientes ( ),Solución de suero al 90\% sin micronutrientes (X), Solución de suero al 90\% con micronutrientes $(\boldsymbol{\Delta})$.

Fuente: Elaboración propia

Los porcentajes de metanización obtenidos demuestran que para concentraciones de 41 y $75 \mathrm{~g} / \mathrm{L}$ de DQO, el lodo anaerobio presenta inhibición en la degradación del suero de queso. Este comportamiento puede relacionarse con las elevadas concentraciones de sodio estimadas en $1900 \mathrm{mg} / \mathrm{L}$ y $3420 \mathrm{mg} / \mathrm{L}$ para las pruebas al 50 y $90 \%$, principalmente en el segundo caso, otros estudios reportan que las concentraciones de sodio que van desde 3500 hasta $5500 \mathrm{mg} / \mathrm{L}$ son moderadamente inhibidores de metanógenos a temperaturas mesofílicas (Kugelman I. y McCarty P., 1964).

\section{Comportamiento del reactor UASB}

El lodo anaerobio inoculado en el reactor UASB tenía una concentración de $42 \mathrm{~g}$ SV/L y una actividad metanogénica de 0,4 g DQO/g SV.d. Este valor pertenece al rango común de 0,1 a 2,0 g DQO/gVS.d reportado en la bibliografía para actividades metanogénicas de lodos anaerobios granulares (Henze M.; Loosdrecht M.; Ekama G.; Brdjanovic D., 2008).

El reactor operó durante 209 días divididos en 4 etapas. En la Fig. 4 se presenta la variación de la VCO y de la eficiencia de remoción de DQO en función del tiempo de operación. Se observa que en los primeros 41 días de trabajo la eficiencia de remoción de DQO presentó amplias fluctuaciones, propias de una fase de arranque y por la tendencia de la alimentación a acidificarse. El día 27 se observó una pronunciada disminución de la eficiencia hasta $36 \%$, debido a que la alcalinidad que se adicionaba a la alimentación no era suficiente para mantener un $\mathrm{pH}$ neutro, con lo que el $\mathrm{pH}$ en el reactor también se redujo de un valor inicial de 7,7 a 6,8. A partir de ese día se suministró $\mathrm{NaHCO}_{3}$ y NaOH constantemente a la alimentación para neutralizarla y con ello se logró mantener el pH dentro del reactor entre 7,4 y 8,4. 
En los días posteriores de la primera etapa, la operación se estabilizó y se alcanzó una eficiencia de eliminación de DQO promedio igual a $83 \%$, trabajando con valores promedio de DQO en la alimentación, VCO y TRH de 1,1 g/L, 2,5 g DQO/L.d y 0,45 días, respectivamente.

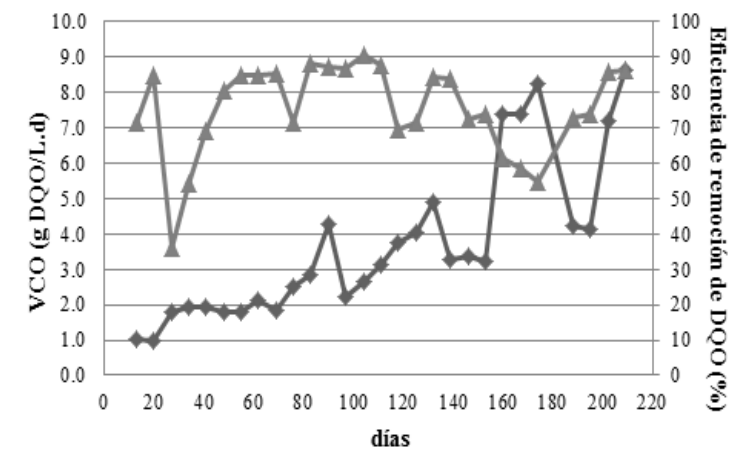

Figura 4. Variación de la Velocidad de Carga Orgánica $(\downarrow)$ y Eficiencia de remoción de DQO ( $\boldsymbol{\Delta})$ vs tiempo.

Fuente: Elaboración Propia

En la segunda etapa de operación, la eficiencia de remoción de DQO varió entre 69 y 91\% (Fig. 4). Los valores promedio de remoción de DQO, DQO en la alimentación, VCO y TRH durante esta etapa fueron $81 \%$, 2,5 g/L, 3,2 g DQO/L.d y 0,79 días, respectivamente.

En la tercera etapa la VCO tuvo un valor promedio de $3.7 \mathrm{~g} \mathrm{DQO} / \mathrm{L} . \mathrm{d}$, el TRH promedio fue 1,7 días y la DQO promedio en la alimentación fue $6,3 \mathrm{~g} / \mathrm{L}$. Bajo estas condiciones la eficiencia de remoción de DQO se mantuvo entre 72 y $85 \%$, con un valor promedio de $77 \%$.

Entre los días 118 y 139, se comprobó que la incorporación de micronutrientes no influía en las eficiencias de remoción de DQO, por lo que se dejó de añadirlos.

En los primeros días de la cuarta etapa (días 159 al 174), el sistema se hizo inestable y la eficiencia de remoción de DQO disminuyó a valores entre 55 y $61 \%$ como consecuencia del incremento de la VCO hasta un valor promedio de 7,7 g DQO/L.d. La posterior disminución de la VCO a valores semejantes a los de la tercera etapa supuso el incremento de la eficiencia (Fig 4.).

Finalmente, con un nuevo incremento de la VCO al valor promedio de 7,9 g DQO/L.d (días 202 al 209) y aplicando un TRH promedio de 1,5 días, la eficiencia de remoción de DQO se mantuvo elevada, alcanzando un valor promedio de $86 \%$. El comportamiento mostrado por el sistema durante la cuarta etapa de experimentación evidencia que la adaptación de la biomasa a las nuevas condiciones de operación (duplicación de la $\mathrm{VCO}$ ), fue relativamente lenta.

Las concentraciones de DQO del suero diluido que se alimentó al reactor UASB se encontraron dentro o próximas al rango usual de DQO de las aguas residuales de la industria láctea, comprendido entre 1 y 9 g/L (Demirel et al., 2005).

Las concentraciones del efluente del reactor UASB en las dos primeras etapas de experimentación estuvieron siempre por debajo de $850 \mathrm{mg} / \mathrm{L}$ y en las etapas 3 y 4 , resultaron por debajo de $2000 \mathrm{mg} / \mathrm{L}$, la mayor parte del tiempo.

En la tabla 7 se comparan los valores promedio de remoción de DQO y parámetros de operación, con los resultados obtenidos por otros autores, sin incluir la fase inicial de arranque ni las bajas eficiencias presentadas dentro de la etapa 4 de experimentación.

Se puede observar que las eficiencias, aunque altas, fueron menores a las mostradas por Yan et al. (1989) y Kalyuzhnyi S., Perez E., Rodriguez J. (1997), quienes trabajaron con concentraciones de DQO mayores y velocidades de carga orgánica semejantes o mayores (Tabla 7). Esta diferencia en las eficiencias de remoción de DQO se atribuye principalmente a los menores TRH aplicados en el presente trabajo, que permitieron una importante degradación de la materia orgánica aunque no en los niveles mostrados por los mencionados autores.

El suero por su contenido en lactosa favorece el desarrollo de bacterias ácido-lácticas, que convierten la lactosa en ácido láctico. Este ácido es usualmente considerado como precursor del ácido propiónico durante la digestión anaerobia, y al ser su metanogénesis más lenta que la del acetato y del butirato, se convierte en la limitante del proceso (Bo Z., Wei-min C., Pin-jing H., 2007). En la literatura, algunos autores consideran que el TRH admisible mínimo para alcanzar la operación estable de reactores anaerobios que tratan suero de queso bruto es 5 días (Kalyuzhnyi et al., 1997, Yan et al. 1989).

Arango y Sanches e Sousa (2009) aplicando valores de DQO y un rango de VCO semejantes al de la presente investigación, obtuvieron eficiencias de remoción de DQO mayores (Tabla 7). Este mejor resultado también estaría relacionado con el empleo de un TRH constante de 1,3 días, a diferencia de los TRH promedio de 0,45 y 0,79 días usados en la primera y segunda etapa del presente trabajo. Sólo en la tercera y cuarta etapa los tiempos de retención hidráulicos promedio fueron $1,75 \mathrm{y}$ 1,5 días, respectivamente.

Los resultados del presente estudio están de acuerdo con lo obtenido por Ramasamy E., Gajalakshmi S., Sanjeevi R., Jithesh M., Abbasi S. (2004) quienes operando un reactor UASB de laboratorio con agua residual láctea sintética a $30 \pm 2{ }^{\circ} \mathrm{C}$, un TRH de 0,5 días y VCO de $2,4 \mathrm{~g}$ DQO/L.d, alcanzaron porcentajes de remoción de DQO entre 73,5 y 85\%. Al incrementar la $\mathrm{VCO}$ a 3,6 g DQO/ L.d las eficiencias se ubicaron entre 82 y $90 \%$, y con un aumento de la VCO hasta 7,2 g DQO/L.d, la eficiencia del proceso se redujo a $50 \%$. 
Tabla7. Comparación de los resultados obtenidos con los de otras investigaciones.

\begin{tabular}{cccccc}
\hline Referencia & $\mathrm{T}\left({ }^{\circ} \mathrm{C}\right)$ & $\begin{array}{c}\mathrm{TRH} \\
(\mathrm{d})\end{array}$ & $\begin{array}{c}\mathrm{DQO} \\
\text { alimentación }\end{array}$ & $\begin{array}{c}\mathrm{VCO} \\
(\mathrm{gDQO} / \mathrm{L} . \mathrm{d})\end{array}$ & $\begin{array}{c}\text { \%Eficiencia remoción } \\
\text { de DQO }\end{array}$ \\
\hline $\begin{array}{c}\text { Yan } \text { et al. }(1989) \\
\text { Kalyuzhnyi }\end{array}$ & $32-34$ & 5 & $4,6-38,1$ & $0,9-7,7$ & $97-99$ \\
$\quad \begin{array}{c}\text { et al. }(1997) \\
\text { Arango y Sanches e } \\
\quad \text { Sousa (2009) }\end{array}$ & Ambiente & 1,3 & $2,3-10,1$ & $1,7-7,5$ & $90-97$ \\
Esta Investigación & 35 & $0,45-1,75$ & $1,1-12,1$ & $2,5-7,9$ & $95,3-97,7$ \\
\hline
\end{tabular}

Fuente: Elaboración Propia

Durante la operación se presentó una continua acidificación de la alimentación, que hizo necesaria la adición de bicarbonato de sodio e hidróxido de sodio para neutralizarla.

La Fig.5 muestra la variación de la alcalinidad parcial de la alimentación y la del efluente del reactor UASB. Se observa que el requerimiento de alcalinidad de la alimentación fue mucho mayor cuando estuvo más concentrada (Etapas 3 y 4) que en las etapas 1 y 2, con menor concentración de suero. Sin embargo, a partir del día 188 (Etapa 4) se pudo trabajar con alcalinidades en la alimentación menores a $5000 \mathrm{mg} \mathrm{CaCO} 3 / \mathrm{L}$ al comprobar que el nivel de alcalinidad que se alcanzaba dentro del reactor era suficiente para mantener el $\mathrm{pH}$ del reactor en 7,5 .

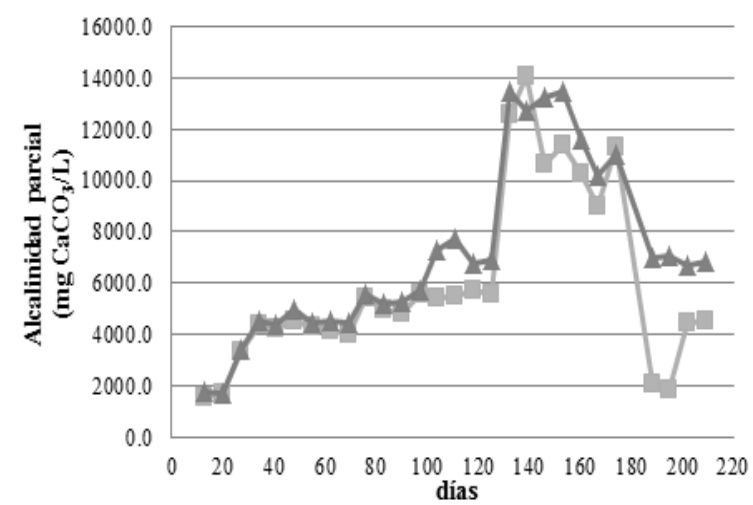

Figura 5. Variación de la alcalinidad parcial de la alimentación ( $\mathbf{\square})$ y del efluente $(\boldsymbol{\Delta})$.

Fuente: Elaboración Propia

Distintos autores han señalado la necesidad de aportar alcalinidad durante el tratamiento anaerobio del suero de queso para lograr el control del $\mathrm{pH}$ y la estabilidad del sistema (Kalyuzhnyi et al., 1997). No obstante, también existen experiencias que expresan que el aporte de alcalinidad puede suspenderse después de un tiempo de operación alrededor de 78 días cuando el TRH de un reactor UASB se mantiene entre 2,5 y 6 días y se utilizan VCOs entre 10 y 28,5 gDQO/L.d (Kalyuzhnyi et al., 1997).
Los datos de alcalinidad intermedia y alcalinidad parcial en el efluente se relacionaron para obtener el índice $\alpha$. La Fig. 6 compara este índice con la eficiencia del proceso, se observa que elevadas eficiencias de remoción de DQO corresponden a valores del índice $\alpha$ generalmente por debajo de 0,3 . Por el contrario, cuando la eficiencia es igual o menor que $50 \%$ el índice $\alpha$ presenta valores mayores que 0,3. Estos resultados están de acuerdo con lo establecido por Ripley et al. (1986) para una digestión anaerobia eficiente. De esta forma, la determinación del índice $\alpha$ se confirma como una herramienta de suma utilidad y de bajo costo en el monitoreo de la degradación anaerobia en reactores UASB.

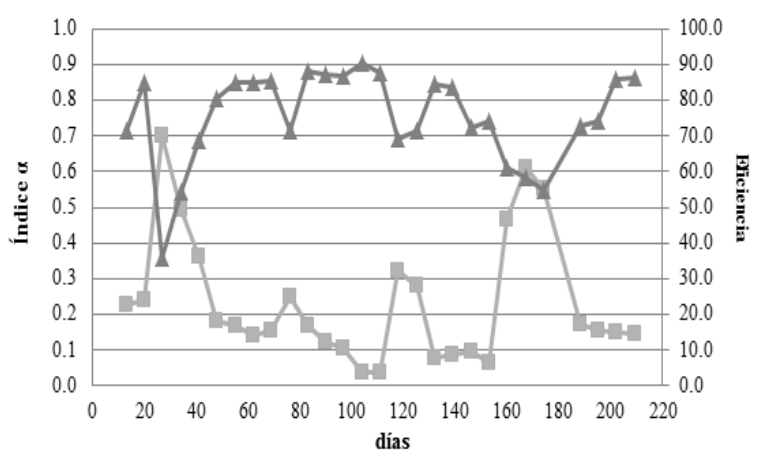

Figura 6. Variación del índice $\alpha$ en el efluente ( $\square$ ) y de la eficiencia del proceso $(\boldsymbol{\Delta})$.

Fuente: Elaboración propia

\section{Conclusiones}

La biodegradación anaerobia del suero de queso (32$35^{\circ} \mathrm{C}$ ) con concentraciones alrededor de $4 \mathrm{~g} \mathrm{DQO} / \mathrm{L}$ no presentó inhibición; por el contrario, sí lo hicieron muestras de suero con concentraciones de 41 y 75 g $\mathrm{DQO} / \mathrm{L}$, comportamiento que se ha asociado con la presencia de altas concentraciones de sodio. La adición de micronutrientes solo favoreció la degradación anaerobia del suero de queso que contenía 41 y 75 g DQO/L. 
El reactor UASB demostró ser un eficiente método de tratamiento de suero de queso diluido $\left(35^{\circ} \mathrm{C}\right)$ para la reducción de DQO. Se alcanzaron porcentajes de remoción promedio de DQO entre 77 y $86 \%$, trabajando con tiempos de residencia hidráulicos entre 0,45 y 1,75 días, velocidades de carga orgánica entre 2,5 y 7,9 g DQO/L.d y DQO en la alimentación entre 1,1 y 12,1 g $\mathrm{DQO} / \mathrm{L}$.

La adición de alcalinidad al suero de queso es fundamental para alcanzar un $\mathrm{pH}$ óptimo para el tratamiento anaerobio y asegurar la estabilidad de sistema. Así también, el control de la relación de alcalinidades IA/PA en el efluente del reactor permite realizar un buen seguimiento del proceso debido a su relación con la eficiencia de remoción de DQO y su capacidad para detectar problemas de acidificación. Valores menores que 0.3 indicaron una buena remoción de DQO.

\section{Literatura citada}

American Public Health Association (APHA). 2005. Standard methods for the examination of water and wastewater. $21^{\text {th }}$ edition.

Arango, O. y Sanches e Sousa, L. 2009. Tratamiento de Aguas Residuales de la Industria Láctea en Sistemas Anaerobios tipo UASB. Facultad de Ciencias Agropecuarias 7 (2): 24-31

Association of official Analytical chemists (AOAC). 1998. Officials methods of Analysis. 18th edition.

Bo, Z., Wei-min, C., Pin-jing, H. 2007. Influence of lactic acid on the two-phase anaerobic digestion of kitchen wastes. Journal of Environmental Sciences 19: 244-249.

Chernicharo, C. A. 2007. Principios del tratamiento biológico de aguas residuales. Vol 5. 1 ra Ed. en español. Editorial Universitaria. Colombia.

Dareioti, M., Dokianakis, S., Stamatelatou, K., Zafiri, C. y Kornaros, M. 2009. Biogas production from anaerobic co-digestion of agroindustrial wastewaters under mesophilic conditions in a two-stage process. Desalination 248: 891-906.

Demirel, B., Yenigun, O. y Onay, T. 2005.Anaerobic treatment of dairy wastewaters: a review. Process Biochemistry 40: 2583-2595.

Ergüder, T., Tezel, U., Güven, E. y Demirer, G. 2001. Anaerobic biotransformation and methane generation potencial of cheese whey in batch and UASB reactors. Waste Management 21(7): 643 -650.

Henze, M., Loosdrecht, M., Ekama, G. y Brdjanovic, D. 2008. Biological Wastewater Treatment. Principles, Modelling and Desing. IWA Publishing. United Kingdom.
Jenkins, S., Morgan, J. y Sawyer, C. 1983. Measuring Anaerobic sludge digestion and growth by a simple alkalimetric titration. Journal Water Pollution Control Federation 55 (5): 448-453.

Kalyuzhnyi, S., Perez, E. y Rodriguez, J. 1997. Anaerobic treatment of high-strength cheese-whey wastewaters in laboratory and pilot uasb-reactors. Biosource Technology 60: 59 -65.

Kugelman, I., y McCarty, P., 1964. Cation toxicity and stimulation in anaerobic waste treatment. Journal Water Pollution Control Federation 37: 97-116.

Mendenhall, W., Beaver, R. y Beaver B. 2006. Introducción a la probabilidad y Estadística. Trad. Brooks/Cole. 12va Edición. México, DF. 776 p.

Mockaitis, G., Ratusznei, S., Rodrigues, J., Zaiat, M. y Foresti, E. 2006. Anaerobic whey treatment by a stirred sequencing batch reactor (ASBR): effects of organic loading and supplemented alkalinity. Journal of Environmental Management 79 (2): 198-206.

Ramasamy, E., Gajalakshmi, S., Sanjeevi, R., Jithesh M. y Abbasi S. 2004. Bioresource Technology 93: 209212

Rico, C., Muñoz, N. y Rico, J. L. 2015. Anaerobic codigestion of cheese whey and the screened liquid fraction of dairy manure in a single continuously stirred tank reactor process: Limits in co-substrate ratios and organic loading rate. Bioresource Technology 189: 327-333.

Ripley, L., Boyle, W. y Converse, J. 1986. Improved alkalimetric monitoring for anaerobic digestion of high-strength wastes. Journal Water Pollution Control Federation 58 (5): 406-411.

Soto, M., Méndez, R. y Lema, J. 1992. Determinación de toxicidad y Biodegradabilidad anaerobia de aguas residuales. Tecnología del Agua no. 92: 70 - 81 .

Superintendencia Nacional de Servicios de Saneamiento (SUNASS). 2013. Las EPS y su desarrollo. Informe 0278-2014/SUNASS-120-F. Lima, Perú.

Yan, J., Lo, K. y Liao, P.1989. Anaerobic Digestion of cheese whey using an upflow anaerobic sludge blanket reactor 27: $289-305$. 